\title{
ON A FINITISTIC COTILTING-TYPE DUALITY
}

\author{
ALBERTO TONOLO
}

\begin{abstract}
Let $R$ and $S$ be arbitrary associative rings. Given a bimodule ${ }_{R} W_{S}$, we denote by $\Delta_{\text {? }}$ and $\Gamma_{\text {? }}$ the functors $\operatorname{Hom}_{?}(-, W)$ and $\operatorname{Ext}_{?}^{1}(-, W)$, where $?=R$ or $S$. We say that ${ }_{R} W_{S}$ is a finitistic weakly cotilting bimodule (briefly FWC) if for each module $M$ cogenerated by $W$, finitely generated or homomorphic image of a finite direct sum of copies of $W, \Gamma M=0=\operatorname{Ext}^{2}(M, W)$. We are able to describe, on a large class of finitely generated modules, the cotilting-type duality induced by a FWC-bimodule.
\end{abstract}

\section{INTRODUCTION}

Let $R$ and $S$ be arbitrary associative rings with unity and ${ }_{R} W_{S}$ be a $R$ - $S$-bimodule. We denote by $\Delta_{R}$ and $\Delta_{S}$ the contravariant functors

$$
\operatorname{Hom}_{R}(-, W): R \text {-Mod } \rightarrow \operatorname{Mod}-S \quad \text { and } \operatorname{Hom}_{S}(-, W): \operatorname{Mod}-S \rightarrow R \text {-Mod }
$$

and by $\Gamma_{R}$ and $\Gamma_{S}$ the contravariant functors

$$
\operatorname{Ext}_{R}^{1}(-, W): R \text {-Mod } \rightarrow \operatorname{Mod}-S \text { and } \operatorname{Ext}_{S}^{1}(-, W): \operatorname{Mod}-S \rightarrow R \text {-Mod. }
$$

In this paper we are interested to describe a cotilting-type duality on the categories of finitely generated modules. The notion of cotilting module appears, in the context of finitely generated modules over artin algebras, as dual of the concept of tilting module, introduced in the early eighties by Brenner and Butler [2] and Happel and Ringel [9]. In 1989 Colby in [3] extended the notion of cotilting to finitely generated modules over noetherian rings which are noetherian over their endomorphism ring. Next, the notion has been once more extended and studied for arbitrary modules over arbitrary rings (see $[4,6,8,5,7]$ ).

In this paper we reconsider the original spirit of the theory concentrating on objects with finiteness conditions, but over arbitrary associative rings. The idea was inspired by reading the paper [1] of Angeleri Hügel. She studied dualities induced by a balanced Colby bimodule ${ }_{R} W_{S}$ (see [1, section 2]), i.e. a bimodule satisfying the following conditions:

1. $\operatorname{Ext}_{?}^{2}(X, W)=0$ for all finitely presented modules $X$,

2. $\operatorname{Ext}_{?}^{1}(W, W)=0$

3. if $\operatorname{Hom}_{?}(X, W)=0=\operatorname{Ext}_{?}^{1}(X, W)$ and $X$ is finitely presented, then $M=0$,

4. ${ }_{R} W$ and $W_{S}$ are finitely generated and the functors $\Delta_{\text {? }}$ carry finitely generated modules to finitely generated modules,

for $?=R$ and $S$. For such a bimodule, the classes $\mathcal{Y}_{\text {? }}$ of finitely generated modules cogenerated by $W$ coincide with the classes of finitely presented modules belonging to $\operatorname{Ker} \Gamma_{\text {? }}$ (see $\left[1\right.$, Proposition 2.6, 4.3]); the functors $\Delta_{\text {? }}$ induce a duality between the classes $\mathcal{Y}_{\text {? }}$ and the functors $\Gamma_{\text {? }}$ induce a duality between the classes $\mathcal{X}_{?}^{\prime}$ of finitely presented modules in Ker $\Delta_{\text {? }}$ (see [1, Theorem 4.4]).

A bimodule ${ }_{R} W_{S}$ is called weakly cotilting (see [10]) if the left $R$ - and right $S$ - modules cogenerated by $W$ are contained in $\operatorname{Ker} \Gamma_{R}$ and $\operatorname{Ker} \Gamma_{S}$, respectively, and the injective dimension of ${ }_{R} W$ and $W_{S}$ is less than or equal to 1 . We say that ${ }_{R} W_{S}$ is a finitistic weakly cotilting bimodule (briefly FWC-bimodule) if for each module $M$ cogenerated by $W$, finitely generated or homomorphic image of a finite direct sum of copies of $W$, we have $\Gamma M=0=\operatorname{Ext}^{2}(M, W)$. For what we have observed before about 
the classes $\mathcal{Y}_{\text {? }}$, any faithfully balanced Colby bimodule ${ }_{R} W_{S}$ is a faithfully balanced FWC-bimodule.

Notation: we denote by $\Delta^{2}$ and by $\Gamma^{2}$ both the compositions $\Delta_{S} \circ \Delta_{R}$ and $\Delta_{R} \circ \Delta_{S}$, and $\Gamma_{S} \circ \Gamma_{R}$ and $\Gamma_{S} \circ \Gamma_{R}$. Throughout the paper, we will write simply $\Delta, \Gamma, \Delta \Gamma$, $\Gamma \Delta, \ldots$ as well "module" when we are indifferently working with left $R$ - or right $S$ modules.

In this paper, using an homological approach similar to [10], we define a natural notion of $\Gamma$-reflexivity (see Definition 2.5). Then we are able to prove a cotilting type duality (see Theorem 2.8) satisfied by a large class of finitely generated modules: in particular, by Proposition 2.9, if ${ }_{R} W_{S}$ is a balanced FWC-bimodule, this class contains all finitely presented modules.

\section{Fixing the Setting}

Let ${ }_{R} W_{S}$ be a bimodule and $\Delta$ and $\Gamma$ be the contravariant functors associated. For each module $M$, we denote by $\delta_{M}: M \rightarrow \Delta^{2} M$ the evaluation map. These maps define natural transformations $\delta$ between the identity functors and $\Delta^{2}$, which are the unities of the right adjoint pair $\left(\Delta_{R}, \Delta_{S}\right)$. A module $M$ is said to be $\Delta$-reflexive if $\delta_{M}$ is an isomorphism. Since the functors $\Gamma_{R}$ and $\Gamma_{S}$ are not adjoint, analogous maps to define the notion of $\Gamma$-reflexivity do not exist. To give a notion of $\Gamma$-reflexivity will be one of the main goals of the next section.

Let $X$ be a module. Consider an exact sequence

$$
0 \rightarrow K \stackrel{\lambda}{\rightarrow} P \stackrel{\varepsilon}{\rightarrow} X \rightarrow 0
$$

with $P$ projective. Applying $\Delta$ two times and considering the evaluation map $\delta$, we obtain the following commutative diagram with exact rows

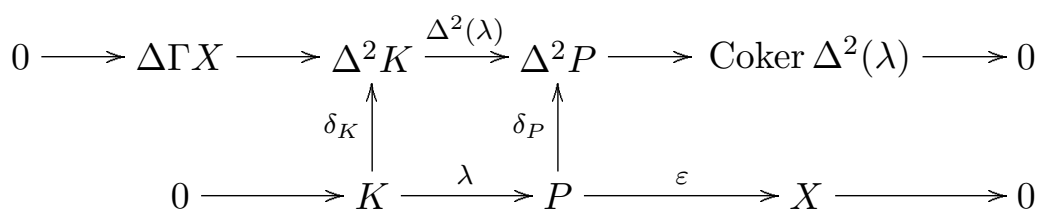

Therefore there exists a unique map $\xi_{P, X}: X \rightarrow$ Coker $\Delta^{2}(\lambda)$ such that coker $\Delta^{2}(\lambda)$ 。 $\delta_{P}=\xi_{P, X} \circ \varepsilon$.

Proposition 1.1. The module Coker $\Delta^{2}(\lambda)$ and the map $\xi_{P, X}$ do not depend on the particular exact sequence $0 \rightarrow K \stackrel{\lambda}{\rightarrow} P \stackrel{\varepsilon}{\rightarrow} X \rightarrow 0$ considered.

Proof. Let us consider another exact sequence $0 \rightarrow H \stackrel{\mu}{\rightarrow} Q \stackrel{\eta}{\rightarrow} X \rightarrow 0$. By the projectivity of $P$ and $Q$ there exist morphisms $f_{i}$ and $g_{i}$ such that the diagram

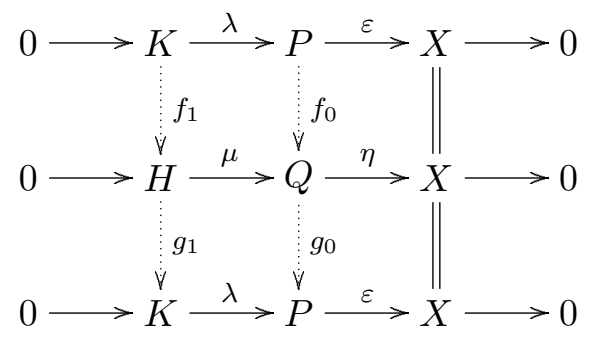

commutes. Denote by $C$ and $C^{\prime}$ the cokernels of $\Delta^{2}(\lambda)$ and $\Delta^{2}(\mu)$, applying the functor $\Delta$ two times, we obtain the following diagram, whose solid part is commutative 
and with exact rows

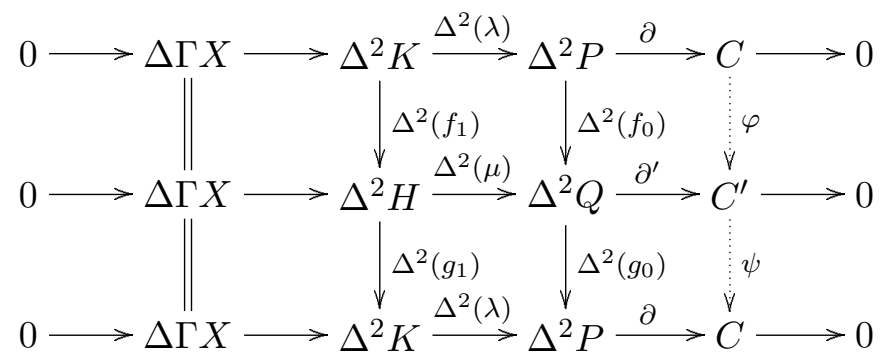

The morphisms $\varphi$ and $\psi$ are the only ones making the diagram commute. We have

$$
\psi \circ \varphi \circ \partial=\partial \circ \Delta^{2}\left(g_{0}\right) \circ \Delta^{2}\left(f_{0}\right)=\partial \circ \Delta^{2}\left(g_{0} \circ f_{0}\right) .
$$

Since there exists $\sigma: P \rightarrow K$ such that $g_{0} \circ f_{0}-1_{P}=\lambda \circ \sigma$, we have $\partial \circ \Delta^{2}\left(g_{0} \circ f_{0}\right)=$ $\partial \circ 1_{\Delta^{2} P}=\partial$ and hence $\psi \circ \varphi=1_{C}$. Analogously we prove $\varphi \circ \psi=1_{C^{\prime}}$ and hence $C$ and $C^{\prime}$ are isomorphic. Moreover, considering the diagram

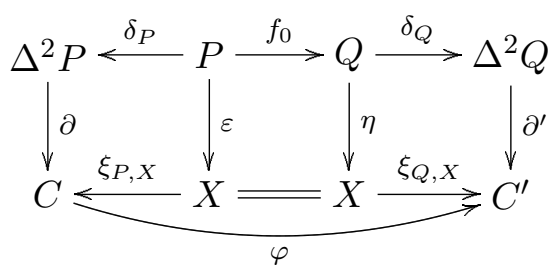

we have

$$
\varphi \circ \xi_{P, X} \circ \varepsilon=\varphi \circ \partial \circ \delta_{P}=\partial^{\prime} \circ \Delta^{2}\left(f_{0}\right) \circ \delta_{P}=\partial^{\prime} \circ \delta_{Q} \circ f_{0}=\xi_{Q, X} \circ \eta \circ f_{0}=\xi_{Q, X} \circ \varepsilon
$$

and hence $\varphi \circ \xi_{P, X}=\xi_{Q, X} ;$ analogously $\psi \circ \xi_{Q, X}=\xi_{P, X}$.

In the sequel the map $\xi_{P, X}$ and the module Coker $\Delta^{2}(\lambda)$ will be dentoted simply with $\xi_{X}$ and $\Xi M$. It is easy to see that for each morphism of modules $f: M \rightarrow N$ there is a uniquely determined morphism $\Xi(f): \Xi M \rightarrow \Xi N$ such that $\Xi(f) \circ \xi_{M}=$ $\xi_{N} \circ f$. Therefore $\Xi$ is a covariant functor and $\xi$ a natural map between $\mathbf{1}$ and $\Xi$. The functor $\Xi$ is not left, nor right, nor half exact, but it preserves epimorphisms. It follows easily by the construction that on the subcategory $\operatorname{Ker} \Delta(\operatorname{resp} . \operatorname{Ker} \Gamma) \Xi$ is a subfunctor of $\Gamma^{2}$ (resp. $\Delta^{2}$ ); we denote by $\kappa$ (resp. $\zeta$ ) the natural embedding between the restrictions to $\operatorname{Ker} \Delta(\operatorname{resp} . \operatorname{Ker} \Gamma)$ of $\Xi$ and $\Gamma^{2}\left(\operatorname{resp} . \Delta^{2}\right)$.

Denote by $\operatorname{gen}(W)$ the class of modules which are homomorphic images of a finite direct sum of copies of $W$.

Definition 1.2. We say that a bimodule ${ }_{R} W_{S}$ is finitistic weakly cotilting (briefly $F W C$-bimodule) if for each module $M$ cogenerated by $W$, finitely generated or in $\operatorname{gen}(W)$, we have $\Gamma M=0=\operatorname{Ext}^{2}(M, W)$.

In the sequel of the paper ${ }_{R} W_{S}$ will be always a FWC-bimodule.

Let $M$ be a finitely generated module. Consider an exact sequence $0 \rightarrow K \rightarrow P \rightarrow$ $M \rightarrow 0$ with $P$ projective and finitely generated. Since $\Delta P$ is a direct summand of $W^{n}, n \in \mathbb{N}, \Gamma \Delta P=0$. In general we know nothing about $\Gamma \Delta K$.

Lemma 1.3. Let $M$ be a finitely generated module. Let us consider the exact sequences $0 \rightarrow H \stackrel{\mu}{\rightarrow} Q \stackrel{\eta}{\rightarrow} M \rightarrow 0$ and $0 \rightarrow K \stackrel{\lambda}{\rightarrow} P \stackrel{\varepsilon}{\rightarrow} M \rightarrow 0$ with $P, Q$ projective and $P$ finitely generated. Then

1. $\Delta^{2}(\eta): \Delta^{2} Q \rightarrow \Delta^{2} M$ is surjective;

2. $\Gamma \Delta K=0$ if and only if, denoting by $J$ the image of $\Delta(\mu)$, the morphism $\partial: \Delta J \rightarrow \Gamma^{2} M$ is surjective. 
Proof. Let us consider the commutative diagram with exact rows

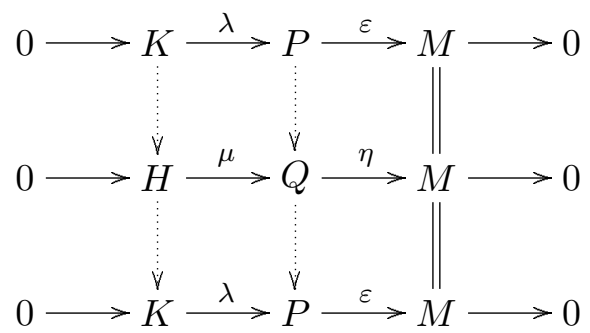

with $P, Q$ projective and $P$ finitely generated. Denote by $I$ and $J$ the images of $\Delta(\lambda)$ and $\Delta(\mu)$. Since $\Delta P$ is a direct summand of $W^{n}, n \in \mathbb{N}$, the module $I$ belongs to $\operatorname{gen}(W)$; since $I \leq \Delta K \in \operatorname{Cogen} W, \Gamma I=0$. Let us apply the functor $\Delta$ two times. 1 follows from the commutativity of the following diagram with exact rows:

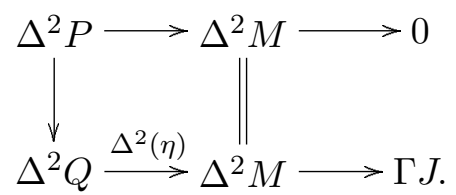

2. Consider the following commutative diagram with exact rows

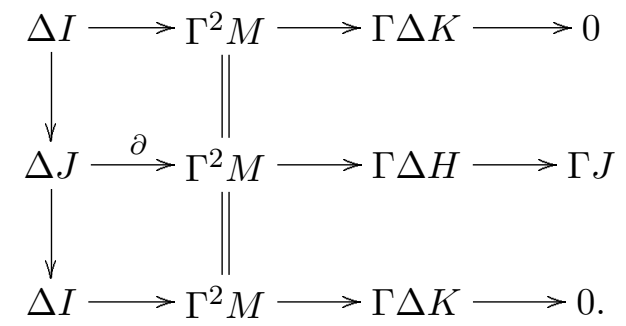

Then $\partial$ is an epimorphism if and only if $\Gamma \Delta K=0$.

We denote by $\mathcal{K}$ the class of finitely generated modules $M$ admitting an exact sequence $0 \rightarrow K \rightarrow P \rightarrow M \rightarrow 0$ with $P$ projective and finitely generated, and with $\Gamma \Delta K=0$. The class $\mathcal{K}$ is not small:

Proposition 1.4. Each finitely presented module $M$ and each finitely generated module $N$ in $\operatorname{Ker} \Gamma$ belong to $\mathcal{K}$.

Proof. Let us consider a projective resolution

$$
\cdots \rightarrow P_{2} \stackrel{d_{1}}{\rightarrow} P_{1} \stackrel{d_{0}}{\rightarrow} P_{0} \stackrel{\varepsilon=d_{-1}}{\rightarrow} M \rightarrow 0
$$

with $P_{0}$ and $P_{1}$ finitely generated. Denote by $K_{i}$ the image of $d_{i}$ and $\lambda_{i}: K_{i} \rightarrow P_{i}$ the morphism induced by $d_{i}$. Let us prove that $\Gamma \Delta K_{0}=0$. We indicate with $I$ the image of $\Delta\left(\lambda_{1}\right)$. Since $\Delta P_{1}$ is a direct summand of a finite direct sum of copies of $W, I \in \operatorname{gen}(W)$ and it is cogenerated by $W$; therefore $\operatorname{Ext}^{2}(I, W)=0$. Applying $\Delta$ to $0 \rightarrow \Delta K_{0} \rightarrow \Delta P_{1} \rightarrow I \rightarrow 0$ we conclude from the exactness of

$$
\Gamma \Delta P_{1}=0 \rightarrow \Gamma \Delta K_{0} \rightarrow \operatorname{Ext}^{2}(I, W)=0 .
$$

If $N$ is finitely generated, there exists an exact sequence $0 \rightarrow H \rightarrow Q \rightarrow N \rightarrow 0$ with $Q$ projective and finitely generated. Since $\Gamma N=0$, applying $\Delta$ we obtain the short exact sequence

$$
0 \rightarrow \Delta N \rightarrow \Delta Q \rightarrow \Delta H \rightarrow 0
$$

Therefore, since $\Delta Q$ is a direct summand of a finite direct sum of copies of $W$, and $\Delta H$ is cogenerated by $W$, we have $\Gamma \Delta H=0$.

The finitely presented modules and the finitely generated modules in $\operatorname{Ker} \Gamma$ are exactly the modules involved in the duality theorem [1, Theorem 4.4]. 
Proposition 1.5. On finitely presented modules the functor $\Xi$ is naturally isomorphic to the 0-th left derived functor $L_{0} \Delta^{2}$.

Proof. Given a finitely presented module $M$, let us consider the projective resolution

$$
\ldots \rightarrow P_{1} \stackrel{d_{0}}{\rightarrow} P_{0} \stackrel{\varepsilon}{\rightarrow} M \rightarrow 0
$$

with $P_{0}$ and $P_{1}$ finitely generated. Denoted by $K_{1}$ the kernel of $d_{0}$ and by $K_{0}$ the kernel of $\varepsilon$, let $\mu_{0}: P_{1} \rightarrow K_{0}$ and $\lambda_{0}: K_{0} \rightarrow P_{0}$ be the morphisms induced by $d_{0}$. By Lemma 1.3, 1., $\Delta^{2}\left(\mu_{0}\right)$ is surjective. Then

$$
\left(L_{0} \Delta^{2}\right) M=\left[\Delta^{2} P_{0}\right] /\left[\operatorname{Im} \Delta^{2}\left(d_{0}\right)\right]=\left[\Delta^{2} P_{0}\right] /\left[\operatorname{Im} \Delta^{2}(\lambda)\right] \cong \operatorname{Coker} \Delta^{2}(\lambda)
$$

Lemma 1.6 (Lemma 1.1, [10]). Given the solid part of the commutative diagram

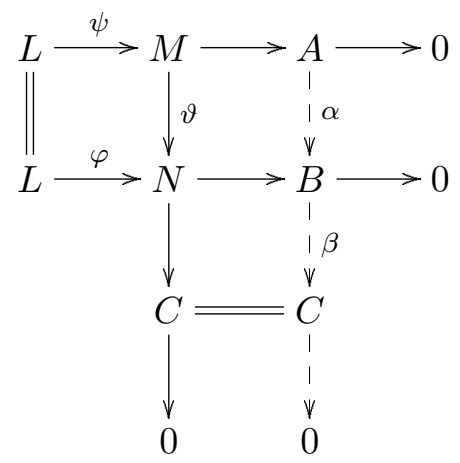

with exact rows and columns, there are unique maps $\alpha$ and $\beta$ such that the diagram commutes. With these maps the second column is exact; moreover, if $\vartheta$ is monic, then so is $\alpha$.

Proof. It follows by diagram chasing.

The following theorem describes the relationships, inside the subcategory $\mathcal{K}$, among the functors $\Gamma^{2}, \Xi$ and $\Delta^{2}$.

Theorem 1.7. There exist, in the subcategory $\mathcal{K}$, natural maps $\alpha$ and $\beta$ between the restrictions of the functors $\Gamma^{2}$ and $\Xi$ and of the functors $\Xi$ and $\Delta^{2}$, such that

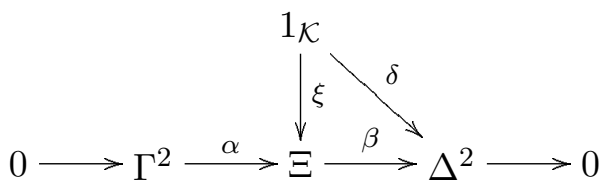

is a commutative diagram with exact row of functors and natural maps. In particular, on the subcategory $\operatorname{Ker} \Gamma_{R} \cap \mathcal{K}$ (resp. $\left.\operatorname{Ker} \Delta_{R} \cap \mathcal{K}\right) \beta$ (resp. $\alpha$ ) is a natural isomorphism.

Proof. Given a module $M \in \mathcal{K}$, let us consider an exact sequence $0 \rightarrow K \stackrel{\lambda}{\rightarrow} P \stackrel{\varepsilon}{\rightarrow}$ $M \rightarrow 0$ with $P$ projective. Denote by $I$ the image of $\Delta(\lambda)$ and by $i: I \rightarrow \Delta K$, $p: \Delta P \rightarrow I$ the morphisms factorizing $\Delta(\lambda)$. Applying the functor $\Delta$ two times we obtain, by Lemma 1.3, after an application of Lemma 1.6, the commutative diagram 
with exact rows and columns

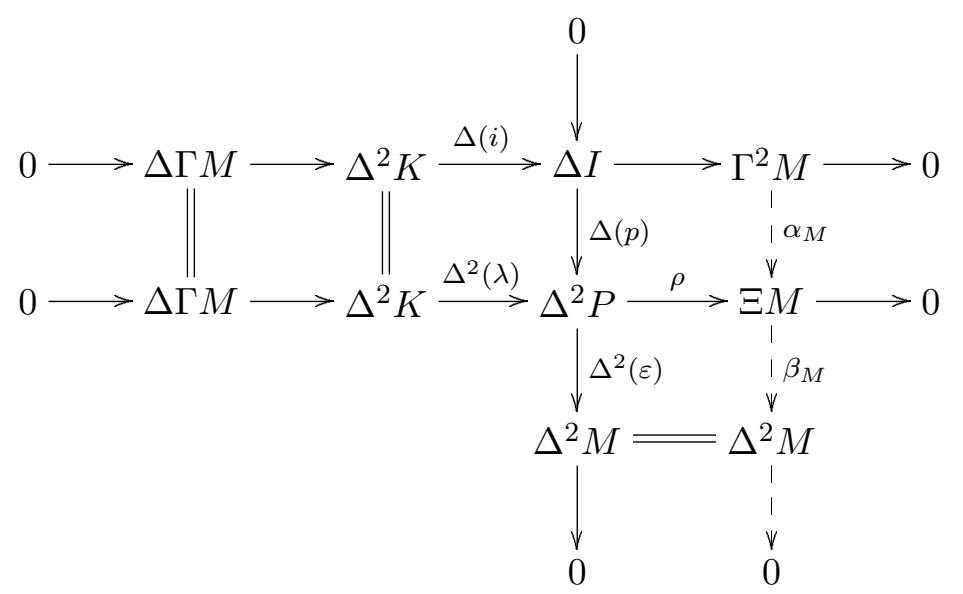

Analogously to the proof of [10, Theorem 1.2], it is possible to prove the naturality of the maps $\alpha_{M}$ and $\beta_{M}$. Finally let us prove that $\beta \circ \xi=\delta$ on $\mathcal{K}$. Looking at the above diagram and at the initial exact sequence $0 \rightarrow K \stackrel{\lambda}{\rightarrow} P \stackrel{\varepsilon}{\rightarrow} M \rightarrow 0$, we have

$$
\beta_{M} \circ \xi_{M} \circ \varepsilon=\beta_{M} \circ \rho \circ \delta_{P}=\Delta^{2}(\varepsilon) \circ \delta_{P}=\delta_{M} \circ \varepsilon .
$$

Then we conclude, since $\varepsilon$ is an epimorphism.

\section{The finitistic Cotilting theorem}

Lemma 2.1. Let us consider, for a finitely generated module $M$, the exact sequence $0 \rightarrow \operatorname{Rej}_{W} M \stackrel{\iota}{\rightarrow} M \stackrel{\pi}{\rightarrow}\left[M / \operatorname{Rej}_{W} M\right] \rightarrow 0$. Then the maps $\Gamma(\iota)$ and $\Delta(\pi)$ are isomorphisms, $\Delta \operatorname{Rej}_{W} M=0$ and $\Gamma\left[M / \operatorname{Rej}_{W} M\right]=0$.

Proof. Applying $\Delta$ to the above exact sequence, we have the long exact sequence

$$
\begin{gathered}
0 \rightarrow \Delta\left[M / \operatorname{Rej}_{W} M\right] \stackrel{\Delta(\pi)}{\rightarrow} \Delta M \rightarrow \Delta \operatorname{Rej}_{W} M \rightarrow \\
\rightarrow \Gamma\left[M / \operatorname{Rej}_{W} M\right] \rightarrow \Gamma M \stackrel{\Gamma(\iota)}{\rightarrow} \Gamma \operatorname{Rej}_{W} M \rightarrow \operatorname{Ext}^{2}\left(\left[M / \operatorname{Rej}_{W} M\right], W\right) .
\end{gathered}
$$

Since $\left[M / \operatorname{Rej}_{W} M\right]$ is finitely generated and cogenerated by $W$, we have

$$
\Gamma\left[M / \operatorname{Rej}_{W} M\right]=0=\operatorname{Ext}^{2}\left(\left[M / \operatorname{Rej}_{W} M\right], W\right) .
$$

Then $\Gamma(\iota)$ is an isomorphism. Since by construction $\Delta(\pi)$ is an isomorphism, $\operatorname{Rej}_{W} M$ belongs to $\operatorname{Ker} \Delta$.

Proposition 2.2. For each $M$ in $\mathcal{K}$, we have the following commutative diagram

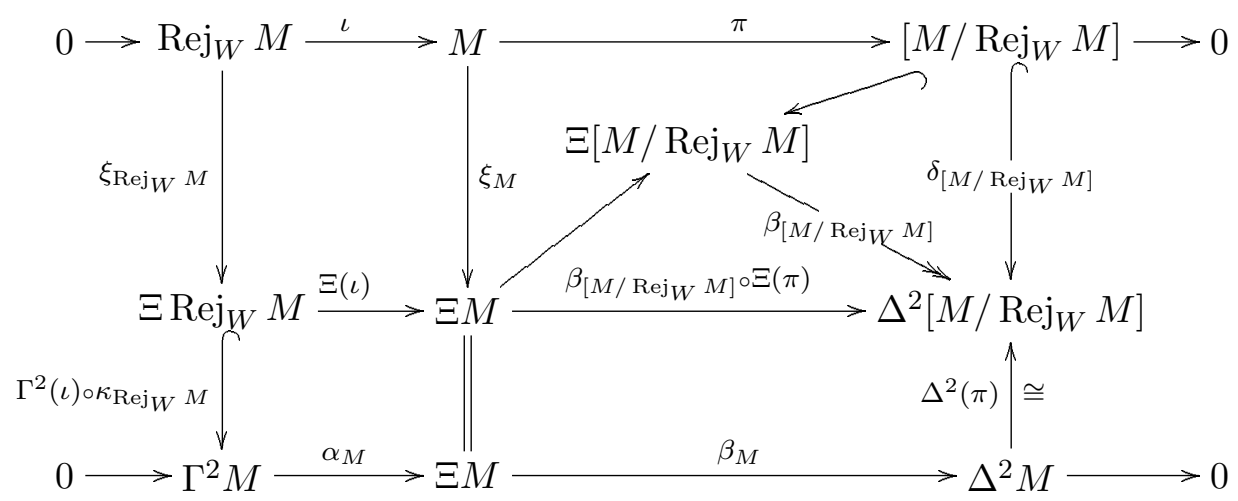

with the first and the third rows exact, $\Xi(\iota)$ injective and $\Xi(\pi)$ surjective.

Proof. First of all, observe that by Proposition $1.4 \mathrm{M} / \operatorname{Rej}_{W} M$ belongs to $\mathcal{K}$ and $\beta_{\left[M / \operatorname{Rej}_{W} M\right]}$ is an isomorphism by Theorem 1.7 and Lemma 2.1. Next, the second row of the diagram is essentially obtained by applying $\Xi$ to the first row; 
since $\Xi$ preserves the epimorphisms, $\Xi(\pi)$ is surjective. By Lemma $2.1, \Delta^{2}(\pi)$ is an isomorphism. Clearly $\delta_{\left[M / \operatorname{Rej}_{W} M\right]}$ is a monomorphism. The third row is the exact sequence obtained in Theorem 1.7. The commutativity of the squares on the top of the diagram follows by the naturality of $\xi$. We have to verify only that $\alpha_{M} \circ \Gamma^{2}(\iota) \circ \kappa_{\operatorname{Rej}_{W} M}=\Xi(\iota)$ and $\Delta^{2}(\pi) \circ \beta_{M}=\beta_{\left[M / \operatorname{Rej}_{W} M\right]} \circ \Xi(\pi)$. Let us prove the first equality; the second one is obtained in a similar way. Consider the commutative diagram with exact rows

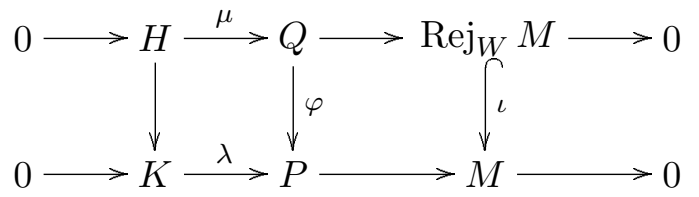

where $Q$ and $P$ are projective, $P$ is finitely generated and $\Gamma \Delta K=0$. Applying $\Delta$, by Lemma 2.1 we get the commutative diagram with exact rows

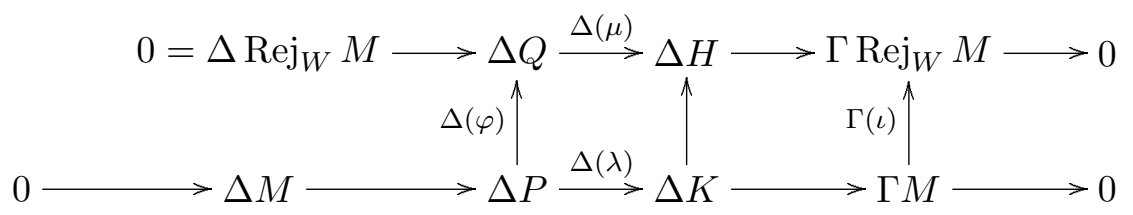

Denote by $I$ the image of $\Delta(\lambda)$, by $p: \Delta P \rightarrow I$ the canonical projection and by $\rho: I \rightarrow \Delta Q$ the induced morphism such that $\rho \circ p=\Delta(\varphi)$. Applying $\Delta$ to the last diagram and $\Xi$ to $0 \rightarrow K \rightarrow P \rightarrow M \rightarrow 0$ we have, proceeding as in the proof of Theorem 1.7, the commutative diagram with exact rows

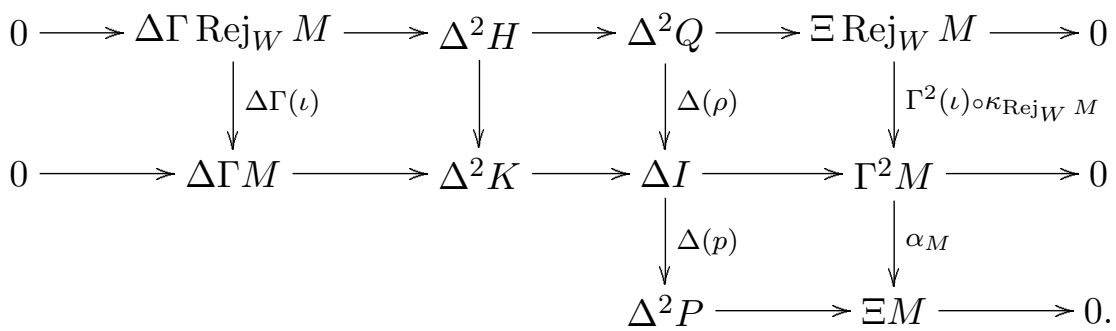

Looking at the first and the third rows of the diagram, since $\Delta(p) \circ \Delta(\rho)=\Delta(\rho \circ$ $p)=\Delta^{2}(\varphi)$, we have $\alpha_{M} \circ \Gamma^{2}(\iota) \circ \kappa_{\operatorname{Rej}_{W} M}=\Xi(\iota)$. In particular, since $\Gamma^{2}(\iota)$ is an isomorphism and $\kappa_{\operatorname{Rej}_{W} M}$ is injective, we obtain that $\Xi(\iota)$ is a monomorphism.

Corollary 2.3. Let $M$ be a module in $\mathcal{K}$. The following assertions are equivalent

1. $\xi_{M}$ is an isomorphism,

2. $\kappa_{\operatorname{Rej}_{W} M}, \xi_{\operatorname{Rej}_{W} M}$ and $\xi_{\left[M / \operatorname{Rej}_{W} M\right]}$ are isomorphisms.

In such a case $\xi_{\Gamma^{2} M}$ and $\xi_{\Delta^{2} M}$ are isomorphisms.

Proof. 1. $\Rightarrow 2$. Clearly $\delta_{\left[M / \operatorname{Rej}_{W} M\right]}$ is an isomorphism. Considering only the first and the third rows of the diagram of Proposition 2.2, by their exactness we have

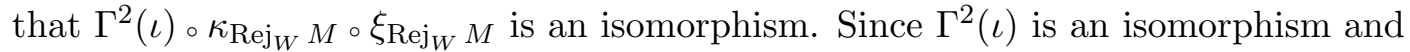
$\kappa_{\operatorname{Rej}_{W}} M$ is a monomorphism, $\kappa_{\operatorname{Rej}_{W}} M$ and $\xi_{\operatorname{Rej}_{W}} M$ are isomorphisms. Finally, since $\xi_{\left[M / \operatorname{Rej}_{W} M\right]} \circ \pi=\Xi(\pi) \circ \xi_{M}$ is an epimorpism, also $\xi_{\left[M / \operatorname{Rej}_{W} M\right]}$ is an isomorphism. 2 . $\Rightarrow 1$. Since $\xi_{\left[M / \operatorname{Rej}_{W} M\right]}$ is an isomorphism, by Theorem 1.7 also $\delta_{\left[M / \operatorname{Rej}_{W} M\right]}$ is an isomorphism. Considering again only the first and the third rows of the diagram of Proposition 2.2, by their exactness $\xi_{M}$ is also an isomorphism.

Proposition 2.4. A module $M$ in $\mathcal{K}$ is $\Delta$-reflexive if and only if $\xi_{M}$ and $\beta_{M}$ are isomorphisms. In such a case $\Gamma M=0$.

Proof. Since $\delta_{M}=\beta_{M} \circ \xi_{M}$ the sufficiency is clear. Suppose $\delta_{M}=\beta_{M} \circ \xi_{M}$ an isomorphism; looking at the diagram of Proposition 2.2, this happen if and only if 
$\delta_{\left[M / \operatorname{Rej}_{W} M\right]} \circ \pi$ is an isomorphism. Now, since $\pi$ is surjective, $\delta_{\left[M / \operatorname{Rej}_{W} M\right]} \circ \pi$ is an isomorphism if and only if both $\pi$ and $\delta_{\left[M / \operatorname{Rej}_{W} M\right]}$ are isomorphisms: in particular, in such a case, $\operatorname{Rej}_{W} M=0$. Then, by Theorem 1.7, $\beta_{\left[M / \operatorname{Rej}_{W} M\right]}=\beta_{M}$ is an isomorphism and hence $\xi_{M}$ is also an isomorphism. Finally, since $M \cong \Delta^{2} M$, it is finitely generated and cogenerated by $W$ : therefore $\Gamma M=0$.

On the subcategory $\operatorname{Ker} \Gamma$, the evaluation map $\delta$ is equal to the composition of the natural map $\xi$ and the natural embedding $\zeta$ between the restrictions of $\Xi$ and $\Delta^{2}$. In particular a module $M$ in $\operatorname{Ker} \Gamma$ is $\Delta$-reflexive if and only if both $\xi_{M}$ and $\zeta_{M}$ are isomorphisms.

Definition 2.5. We say that a module $M$ in $\operatorname{Ker} \Delta$ is $\Gamma$-reflexive if and only if $\xi_{M}$ and $\kappa_{M}$ are isomorphisms.

Proposition 2.6. A module $M$ in $\mathcal{K}$ belongs to $\operatorname{Ker} \Delta$ and it is $\Gamma$-reflexive if and only if $\xi_{M}$ and $\alpha_{M}$ are isomorphisms.

Proof. It is an easy consequence of Proposition 2.2.

Let us denote by $\mathcal{M}$ the classes of left $R$ - and right $S$ - modules in $\mathcal{K}$ such that $\xi_{M}$ is an isomorphism.

For each module $M$ in $\mathcal{M}$ we define a morphism $\gamma_{M}: \Gamma^{2} M \rightarrow M$, setting $\gamma_{M}=$ $\xi_{M}^{-1} \circ \alpha_{M}$. It is a natural transformation between $\Gamma^{2}$ and the identity functor restricted to $\mathcal{M}$. The module $M$ is $\Gamma$-reflexive if and only if $\gamma_{M}$ is an isomorphism.

Lemma 2.7. Let $M$ be a module in $\mathcal{M}$. If $\Delta \Gamma M=0$, then $\Gamma\left(\alpha_{M}\right)$ is an isomorphism and

$$
\Gamma\left(\xi_{M}\right) \circ\left[\Gamma\left(\alpha_{M}\right)\right]^{-1} \circ \kappa_{\Gamma M} \circ \xi_{\Gamma M}=1_{\Gamma M} .
$$

Proof. Since $M \cong \Xi M$ are finitely generated, $\Delta^{2} M$ is finitely generated and cogenerated by $W$ : thus, since $\Gamma \Delta^{2} M=0, \Gamma\left(\alpha_{M}\right)$ is an isomorphism. Next, consider a short exact sequence $0 \rightarrow K \stackrel{i}{\rightarrow} P \stackrel{p}{\rightarrow} M \rightarrow 0$ with $P$ finitely generated and projective, and $\Gamma \Delta K=0$. Denote by $I$ the image of $\Delta(i)$, we have the following commutative diagram with exact rows:

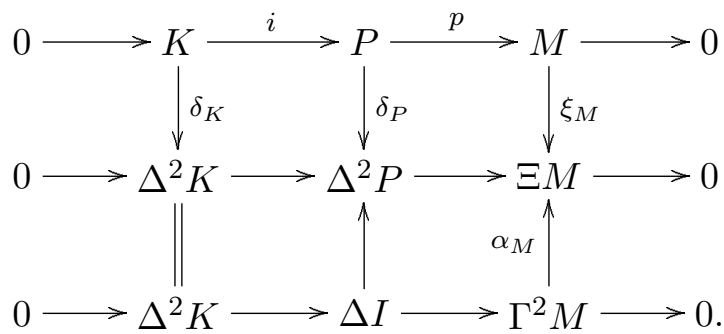


Applying $\Delta$ to it and $\Xi$ to $\Delta K \stackrel{\partial_{1}}{\rightarrow} \Gamma M \rightarrow 0$ we get the following diagram

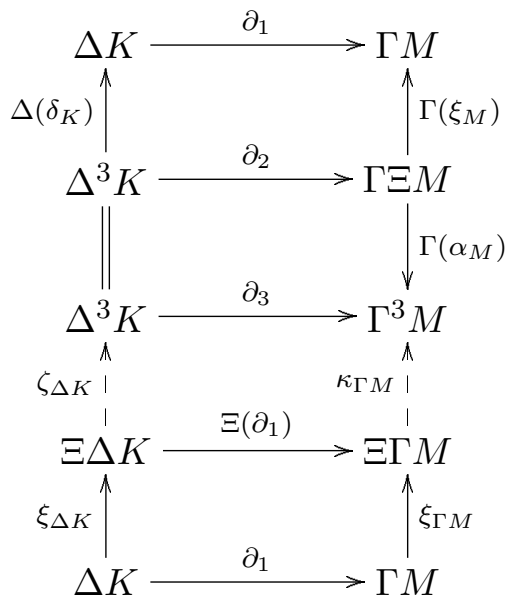

Its solid part is commutative; let us prove that the whole diagram is commutative. Given an exact sequence $0 \rightarrow H_{1} \rightarrow Q \stackrel{q}{\rightarrow} \Delta K \rightarrow 0$ with $Q$ projective, we can construct the following commutative diagram with exact rows

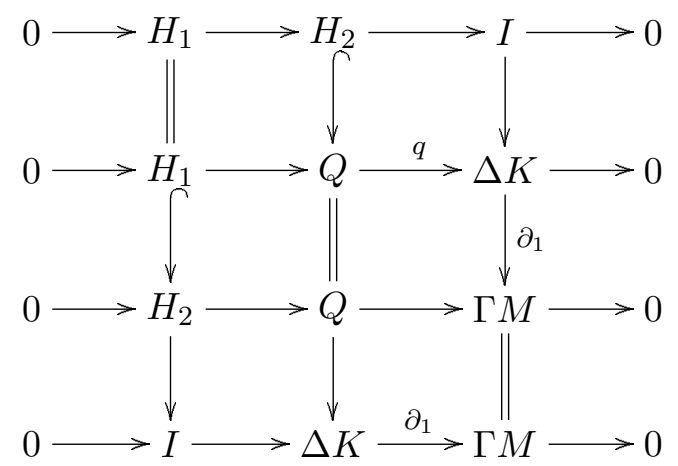

where $H_{2}=q^{-1}(I)$. Since $I \in \operatorname{gen}(W)$ and it is cogenerated by $W, \Gamma I=0$. We have also $\Delta \Gamma^{2} M=0$ : indeed, looking at the diagram of Proposition 2.2, since $\xi_{M}$ is an isomorphism we have that $\delta_{M / \operatorname{Rej}_{W} M}$ is an isomorphism; then, since $\Gamma^{2}(\iota)$ is an isomorphism, by diagram chasing we get that $\xi_{\operatorname{Rej}_{W} M}$ and $\kappa_{\operatorname{Rej}_{W}} M$ are isomorphisms, and hence $\Gamma^{2} M \cong \Gamma^{2} \operatorname{Rej}_{W} M \cong \operatorname{Rej}_{W} M$ belong to Ker $\Delta$. Therefore, applying $\Delta$ two times we have the following commutative diagram with exact rows

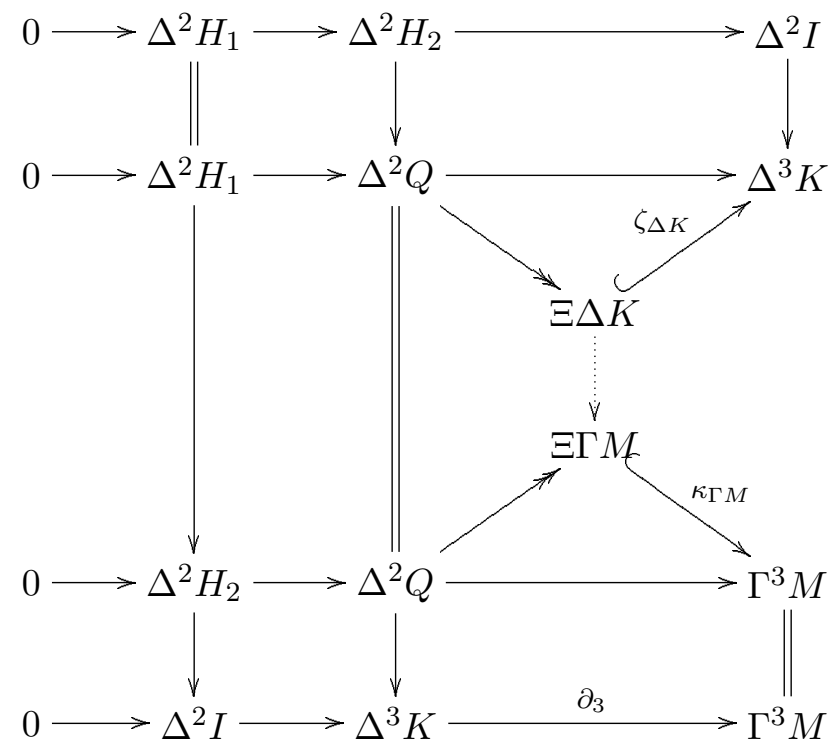


The dotted arrow $\Xi \Delta K>-\exists \Gamma M$ is the only one such that the diagram commutes. On one side it is, by construction, $\Xi\left(\partial_{1}\right)$; on the other side, looking at the commutative right bottom square, it must be the restriction of $\partial_{3}$ to $\Xi \Delta K$ and $\Xi \Gamma M$. Therefore, the whole diagram (\#) commutes. Now the requested identity follows by

$$
\begin{gathered}
\Gamma\left(\xi_{M}\right) \circ\left[\Gamma\left(\alpha_{M}\right)\right]^{-1} \circ \kappa_{\Gamma_{M}} \circ \xi_{\Gamma M} \circ \partial_{1}=\Gamma\left(\xi_{M}\right) \circ\left[\Gamma\left(\alpha_{M}\right)\right]^{-1} \circ \kappa_{\Gamma_{M}} \circ \Xi\left(\partial_{1}\right) \circ \xi_{\Delta K}= \\
=\Gamma\left(\xi_{M}\right) \circ\left[\Gamma\left(\alpha_{M}\right)\right]^{-1} \circ \partial_{3} \circ \zeta_{\Delta K} \circ \xi_{\Delta K}=\Gamma\left(\xi_{M}\right) \circ \partial_{2} \circ \zeta_{\Delta K} \circ \xi_{\Delta K}= \\
=\partial_{1} \circ \Delta\left(\delta_{K}\right) \circ \zeta_{\Delta K} \circ \xi_{\Delta K}=\partial_{1} \circ \Delta\left(\delta_{K}\right) \circ \delta_{\Delta K}=\partial_{1}
\end{gathered}
$$

and the fact that $\partial_{1}$ is epic.

Theorem 2.8 (Cotilting Theorem). For each module $M \in \mathcal{M}$ the sequence

$$
\text { (*) } \quad 0 \rightarrow \Gamma^{2} M \stackrel{\gamma_{M}}{\rightarrow} M \stackrel{\delta_{M}}{\rightarrow} \Delta^{2} M \rightarrow 0
$$

is exact, $\Delta M$ and $\Delta^{2} M$ are $\Delta$-reflexive and $\Gamma^{2} M$ is $\Gamma$-reflexive. If $\Delta \Gamma M=0$, then also $\Gamma M$ is $\Gamma$-reflexive.

Proof. Since $\xi_{M}: M \rightarrow \Xi M$ is an isomorphism, and $\beta_{M \circ} \circ \xi_{M}=\delta_{M}$ and $\xi_{M}^{-1} \circ \alpha_{M}=$ $\gamma_{M}$, we obtain the sequence $(*)$ from Theorem 1.7. Looking at Proposition 2.2, we have that $\delta_{\left[M / \operatorname{Rej}_{W} M\right]}$ is an isomorphism and hence $\Delta^{2} M \cong\left[M / \operatorname{Rej}_{W} M\right]$ is $\Delta$ reflexive. Analogously, as observed in the proof of Lemma 2.7 , we get that $\Gamma^{2} M \cong$ $\operatorname{Rej}_{W} M$ is $\Gamma$-reflexive. In particular $\Delta \Gamma^{2} M=0$; therefore, applying $\Delta$ to the exact sequence $(*)$, we obtain that $\Delta\left(\delta_{M}\right)$ is an isomorphism. Since the natural maps $\delta$ are the unities of the adjunction between the functors $\Delta_{R}$ and $\Delta_{S}$, we have that $\delta_{\Delta M}$ is an isomorphism and hence $\Delta M$ is $\Delta$-reflexive. Finally, suppose $\Delta \Gamma M=0$. By Lemma 2.7, $\kappa_{\Gamma M} \circ \xi_{\Gamma M}$ is an isomorphism. Since $\kappa_{\Gamma M}$ is injective, both $\kappa_{\Gamma M}$ and $\xi_{\Gamma M}$ are isomorphisms.

Proposition 2.9. If the $F W C$-bimodule ${ }_{R} W_{S}$ is balanced, then the finitely presented modules belong to $\mathcal{M} \cap \operatorname{Ker}(\Delta \Gamma)$; a finitely generated module $M$, such that $\Gamma M=0$, belongs to $\mathcal{M} \cap \operatorname{Ker}(\Delta \Gamma)$ if and only if it is cogenerated by $W$.

Proof. Let $M$ be a finitely presented module. In Proposition 1.4 we have seen that any finitely presented module $M$ belongs to $\mathcal{K}$. We have to prove that $\xi_{M}$ is an isomorphism and that $\Delta \Gamma M=0$. Consider an exact sequence $0 \rightarrow K \stackrel{\lambda}{\rightarrow} P \rightarrow M \rightarrow 0$ with $P$ projective and finitely generated and $\Gamma \Delta K=0$. Since $M$ is finitely presented, $K$ is finitely generated. Since the rings $R$ and $S$ are $\Delta$-reflexive, $\delta_{K}$ is surjective and the module $P$ is $\Delta$-reflexive. Therefore, by the following commutative diagram with exact rows

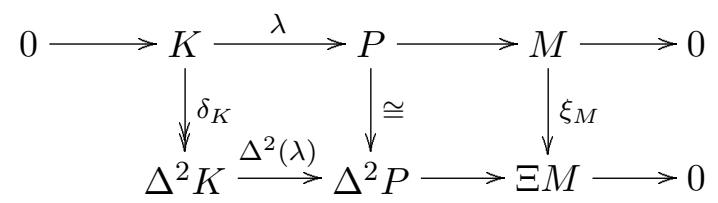

we obtain that $\xi_{M}$ and $\delta_{K}$ are isomorphisms, and $\Delta^{2}(\lambda)$ is injective. Applying $\Delta$ to $0 \rightarrow K \rightarrow P \rightarrow M \rightarrow 0$ we have exact sequences

$$
0 \rightarrow \Delta M \rightarrow \Delta P \rightarrow C \rightarrow 0 \quad \text { and } \quad 0 \rightarrow C \rightarrow \Delta K \rightarrow \Gamma M \rightarrow 0 ;
$$

and applying $\Delta$ again we obtain exact sequences

$$
0 \rightarrow \Delta C \stackrel{\varphi}{\rightarrow} \Delta^{2} P \rightarrow \Delta^{2} M \quad \text { and } \quad 0 \rightarrow \Delta \Gamma M \rightarrow \Delta^{2} K \stackrel{\psi}{\rightarrow} \Delta C .
$$

Since $\varphi \circ \psi=\Delta^{2}(\lambda)$, we have $\Delta \Gamma M=0$.

If $M$ is finitely generated, then $M$ is an homomorphic image of a $\Delta$-reflexive module and hence $\delta_{M}$ is surjective. If $M$ is also cogenerated by $W$, then $\delta_{M}$ is an isomorphism. Since $\Gamma M=0$, by Proposition $1.4 M$ belongs to $\mathcal{K}$ : therefore, by Proposition $2.4, \xi_{M}$ is an isomorphism. Conversely, if $M$ is finitely generated and $\Gamma M=0$ and $\xi_{M}$ is an 
isomorphism, by Proposition 2.2 we have $\operatorname{Rej}_{W} M=0$ and hence $M$ is cogenerated by $W$.

\section{REFERENCES}

[1] L. Angeleri Hügel, Finitely cotilting modules, to appear in Comm. Algebra.

[2] S. Brenner, M. Butler, Generalizations of the Bernstein-Gelfand-Ponomarev reflection functors, in Proceedings ICRA II, Ottawa 1979, LNM 832, (1980), 103-169.

[3] R. Colby, A generalization of Morita duality and the tilting theorem, Comm. Algebra 17(7) (1989), 1709-1722.

[4] R. Colby, A cotilting theorem for rings, Methods in Module Theory, M. Dekker New York 1993, 33-37.

[5] R. Colpi, Cotilting bimodules and their dualities, preprint.

[6] R. Colpi, G. D'Este, A. Tonolo, Quasi-tilting modules and counter equivalences, J. Algebra 191 (1997), 461-494.

[7] R. Colpi, K. Fuller, Cotilting modules and bimodules, to appear in Pacific J. Math.

[8] R. Colpi, A. Tonolo, J. Trlifaj, Partial cotilting modules and the lattices induced by them, Comm. Algebra 25 (1997), 3225-3237.

[9] D. Happel, C. M. Ringel Tilted algebras, Trans. Amer. Math. Soc. 274, (1982), 399-443.

[10] A. Tonolo, Generalizing Morita duality: a homological approach, preprint 1999.

Dipartimento di Matematica Pura ed Applicata, Università di Padova - Italy

E-mail address: tonolo@math.unipd.it 